\title{
Probiotics and prebiotics in atopic dermatitis: Pros and cons (Review)
}

\author{
IONUT SERBAN FANFARET ${ }^{1 *}$, DANIEL BODA $^{2,3}$, LAURA MIHAELA ION $^{3}$, DANIELA HOSSEYNI $^{4}$, \\ POLIANA LERU ${ }^{5}$, SELDA ALI $^{6,7^{*}}$, SABINA CORCEA $^{6 *}$ and ROXANA BUMBACEA ${ }^{6,7}$ \\ ${ }^{1}$ Pediatrics Department, Béziers Hospital Center, 34500 Béziers, France; ${ }^{2}$ Dermatology Department, \\ 'Carol Davila' University of Medicine and Pharmacy, 050474 Bucharest; ${ }^{3}$ Pediatrics Department, \\ 'Ponderas' Academic Hospital, 014142 Bucharest, Romania; ${ }^{4}$ Public Health Department, \\ Harvard T.H. Chan School of Public Health, ECPE, PPCR Program, Boston, MA 02115, USA; \\ ${ }^{5}$ Internal Medicine, ${ }^{6}$ Allergy Department, 'Carol Davila' University of Medicine and Pharmacy, 050474 Bucharest; \\ ${ }^{7}$ Allergy Department, 'Dr. Carol Davila’ Clinical Nephrology Hospital, 010731 Bucharest, Romania
}

Received July 16, 2021; Accepted August 16, 2021

DOI: $10.3892 /$ etm.2021.10811

\begin{abstract}
Atopic dermatitis (AD) represents a chronic inflammatory skin condition in which the skin barrier is impaired; thus, the permeability is increased. Hence, there is a greater risk of allergic sensitization, as well as a higher $\mathrm{pH}$ and lower protection against resident microbes. Since this condition is currently increasing among children, it requires further study, as little is known regarding the pathogenesis that makes the skin prone to chronic relapsing inflammation. Trying to standardize the data regarding the use of prebiotics and probiotics in $\mathrm{AD}$, we encountered tremendous variability in the literature data. Literature abounds in conflicting data: studies regarding prophylactic and therapeutic applications, different types of strains and dosages, applications in young children up to 5 years of age and above, usage of probiotics alone, prebiotics alone or synbiotics combined. There are also conflicting data regarding the outcome of these studies; some confirming a positive effect of prebiotics, probiotics or synbiotics and some showing no efficacy at all. The articles were divided into those assessing probiotics or prebiotics alone
\end{abstract}

Correspondence to: Dr Daniel Boda, Dermatology Department, 'Carol Davila' University of Medicine and Pharmacy, 8 Eroilor Sanitari Boulevard, 050474 Bucharest, Romania

E-mail: danielboda.db@gmail.com

Dr Laura Mihaela Ion, Pediatrics Department, 'Ponderas' Academic Hospital, 85a Nicolae G. Caramfil Street, 014142 Bucharest, Romania

E-mail: laura.ion@reginamaria.ro

${ }^{*}$ Contributed equally

Key words: atopic dermatitis, prebiotics, probiotics, synbiotics, gut microbiome, chronic ski inflammation and a combination of the two, with studies showing a positive effect and studies proving no efficacy at all. We tried to critically analyze those articles showing weak and strong points. In summary, the most studied probiotics were the strains of Lactobacilli and Bifidobacteria. The Severity Scoring of Atopic Dermatitis (SCORAD) index was used to measure the efficacy of the treatment. Most studies compared their results with a placebo group and the efficacy when seen in moderate to severe forms of AD in patients with other allergic diseases present. However, the results are difficult to interpret, as in many studies the authors suggest that the disease may have a tendency to improve in time in some groups of patients.

\section{Contents}

1. Introduction

2. Probiotics and prebiotics

3. The microbiome

4. Controversies

5. Conclusions

\section{Introduction}

Atopic dermatitis (AD) represents a chronic inflammatory skin condition in which the skin barrier is impaired; thus, the permeability is increased. Hence, there is a greater risk of allergic sensitization, as well as a higher $\mathrm{pH}$ and lower protection against resident microbes. Since this condition is currently increasing among children, it requires further study, because little is known regarding the pathogenesis that makes the skin prone to chronic relapsing inflammation (1).

$\mathrm{AD}$ is a chronic pruritic, remitting, relapsing inflammatory skin disorder. It is usually related with different symptoms of immunoglobulin E (IgE)-associated allergy, such as allergic rhinoconjunctivitis, allergic asthma and IgE-mediated food allergy. Often, it is the first phase in the sequential development 
of other different atopic conditions, being called the 'atopic march', which includes urticaria, chronic allergic rhinitis, chronic allergic conjunctivitis and asthma (2-4).

Atopic conditions have been on the rise during the past few decades, particularly in industrialized countries. Thus, it seems that the modern lifestyle represents one of the main contributing factors to this worldwide epidemic $(5,6)$.

Different studies show different data, but there is consensus between epidemiological data that approximately $20 \%$ of children are affected by $\mathrm{AD}$, both in developed and developing countries (7).

With roughly $60 \%$ of cases occurring between 0 and 6 months, AD generally manifests in the first 5 years of life $(8,9)$.

Environmental factors, genetics and immunologic markers are some of the elements responsible for the development of AD (10).

There is also an agreement regarding the link between digestive allergens and food diversification in the onset of AD in children up to 5 years of age, whereas in children above 5 years of age respiratory allergies predominate $(11,12)$.

The gastrointestinal tract of a newborn is sterile at birth; the developing microflora in the early postnatal period is involved in the activation of innate and adaptive immunity (13). The development of atopy occurs when inadequate microbial stimulus leads to unbalanced gut microflora, favoring the persistence of a neonatal Th2-dominant immune response $(14,15)$.

The consumption of sterile food, proper hygiene, reduced family size, as well as the increased use of antimicrobial medication have resulted in lower rates of infection during childhood; this reducing early contact to microbes $(16,17)$. This may interfere with the development of the child's immune system, which tends to be directed towards a T-helper (Th)2 phenotype in infants, while postnatal maturation is linked with gradual inhibition of Th2 and increasing Th1 affinity (18-20). The hypothesis that early microbial exposure is a key feature for Th1-skewed immune response in healthy children during the postnatal period is strongly supported by epidemiologic and experimental data $(21,22)$.

There is great interest in the role of microbial products such as probiotics in the prevention and treatment of allergic disease, due to the growing concern regarding the adverse immunologic effects of increasingly more hygienic environments (23).

Positive challenges, such as the exposure of infants to daycare environments and pets, various nonpathogenic bacteria, which may enhance protective immunity against allergic disorders, is beneficial (24).

In patients with $\mathrm{AD}$, skin damage is probably caused mainly by aberrant Th2-type immune responses, resulting in overproduction of proinflammatory cytokines against common environmental allergens $(25,26)$.

Imbalances in certain gut bacterial species are associated with atopic disorders, according to the results of decades of research (27). Despite the fact that the importance of dysbiosis in the gut microbiota in patients with AD has been stressed by these studies, the specific microbial dysfunction that adversely affects the regulation of inflammation underlying AD remains unknown $(28,29)$.

Several grading systems have been used to assess AD, the most common being the SCORAD severity score (Severity
Scoring of Atopic Dermatitis) (30) and IGA (Iinvestigator Global Assessment).

In preparation of this review, we searched the Web of Science database regarding articles published in the last 10 years, using key words such as probiotics, prebiotics, synbiotics, skin microbiome, and atopic dermatitis. Sponsored articles or articles whose authors had relevant conflict of interest were excluded. A total of 63 relevant original articles and reviews were critically reviewed.

\section{Probiotics and prebiotics}

Probiotics are living microorganisms, which may provide positive health benefits by boosting the gastrointestinal microbiome and by regulating the Th1 and Th2 immune system response $(31,32)$.

Nonetheless, the definition of a probiotic cannot elucidate what type of potential health benefits it grants. In addition, not all probiotics influence the immune system in the same manner (33).

By inhibiting the T-helper cell type-2 (Th2)-mediated response and by improving the Th1/Th2 ratio, probiotics can reduce the severity of AD (34). When inhibiting the Th2 cell response, cytokines such as interleukin (IL)-4, IL-5, IL-6 and IL-13 are no longer released, interferon (INF)- $\gamma$ decreases (cytokine released by Th1 cells), phagocytosis is stimulated, and serum $\operatorname{IgA}$ is increased (35).

Microorganisms should comply with the majority, if not all, of the following criteria to qualify as a probiotic: identification at genus, species and strain level, being safe for food and clinical use, being able to survive intestinal transit, being able to adhere to mucosal surfaces, being able to colonize the human intestine or vagina (at least temporarily), producing antimicrobial substances, being able to antagonize pathogenic bacteria, possessing clinically documented and validated health effects and being stable during processing and storage (36).

Probiotics belong to the Lactobacillus group (L. rhamnosus $\mathrm{GG}$, L. sporogenes, L. reuteri RC-14, L. plantarum 299v, L. acidophilus, L. lactis). Bifidobacterium group (B. bifidum, B. longum, B. infantis), Streptococcus group (S. thermophillus, S. lactis, S. fecalis); in addition, there are non-bacterial organisms (non-pathogenic yeast Saccharomyces boulardii). Bifidobacterium and Lactobacillus are a part of the regular microbial flora. These represent Gram-positive, anaerobic types of bacteria. Some types produce both lactic acid and other antimicrobial substances, such as hydrogen peroxide and bacteriocins (small proteins with potential bactericidal effect) (37).

Prebiotics represent selectively fermented dietary ingredients, which promote particular alterations in the composition and/or activity of the gastrointestinal microbiota, with benefits for the health of the host (38). They are indigestible substances that affect the host in a beneficial manner by selectively stimulating the growth and/or the activity of a limited number of bacterial strains already established in the gut flora (39). Prebiotics are nondigestible oligosaccharides in general and fructooligosaccharides in particular. They seem to stimulate the growth of endogenous bifidobacterial (40).

Prebiotics increase the production of short-chain fatty acids (SCFAs) (acetate, propionate and butyrate) with 
anti-inflammatory effects (41), decrease the generation of toxic fermentation products (42) and enhance the Th1/Th2 ratio, increase lymphocyte and/or leukocyte numbers in gut-associated lymphoid tissues and increase intestinal IgA secretion (43).

Consequently, a food ingredient is categorized as prebiotic when it meets three criteria: firstly, it escapes digestion in the upper gastrointestinal tract and reaches the colon intact; secondly, it ferments due to the intestinal microflora. Third, it should stimulate the growth of intestinal bacteria linked to wellbeing and health in a selective manner (44-47).

\section{The microbiome}

The microbiome represents a set of microorganisms (bacteria, fungi, archaebacteria and protozoa) and viruses, which colonize a specific environment. Physiologically, and sometimes pathologically, they co-exist in a symbiotic relationship with the human body (48).

New scientific findings have demerge from the study of the human microbiome. Currently, there is a wide range of available data: a vast database of bacterial species isolated from many body areas is currently available to scientists. Their task is to pinpoint any dysbiosis potentially responsible for allergic diseases (48).

The gut microbiome represents a key postnatal immune regulator, which promotes the immune maturation of Th1 and Treg lymphocyte functions and suppresses the Th2 response, prevalent during the fetal period. Dysbiosis, intended as a dysregulation of the microbiome, particularly if present in the neonatal period, can be a cofactor in the genesis of allergic disorders, because of its role in the disruption of immune maturation (49).

The absence of antibiotic therapies in the early years of life, vaginal delivery, exclusive breastfeeding for the first 4 months, the presence of pets at home during infancy or pregnancy, the absence of maternal antibiotic therapies during pregnancy are some of the elements that appear to result in the establishment of a protective microbiome against allergic disorders. All of them are actually linked to lower rates of childhood allergies (50).

The research regarding newborns delivered either vaginally or by Caesarean section showed that various areas (mouth, skin, intestines) are colonized by species like Sneathia and Lactobacillus spp. (bacteria present in the maternal genital tract). Children delivered by Caesarean section display a widespread of Staphylococcus and Streptococcus spp. These results are in accordance with previous epidemiological studies that show a low risk of developing allergic disorders in newborns delivered vaginally (51).

According to the diet-microbiome hypothesis, changes in the Westernized diet that reflect a lower intake of fiber may result in alterations in the gut microbiome, followed by decreased production of immunomodulatory products, particularly SCFAs, which have anti-inflammatory effects and contribute to the maintenance of epithelial barrier function (52).

There are studies that have reported a lower incidence of asthma in children living in a rural area compared to those living in urban areas. The exposure to animals, in particular, plays a protective role against bronchial hyperreactivity. This does not occur only in children, but also during the fetal period; the children of mothers who spend their pregnancies in rural areas have less chances of developing asthma; this suggests a modulation of the fetal immune system by the microbiome (53).

Studies regarding gut infections caused by $C$. difficile have described the role of the dysregulation of the gut microbiome following antibiotic therapies; the growth of this bacterium and the resulting stage of infection have been effectively inhibited through the use of fecal transplant in order to correct the gut dysbiosis. This therapeutic success supports the finding of microorganisms (also called probiotics) or substances that can be metabolized and contribute to the growth of some bacterial species (prebiotics) used to treat other states of dysbiosis that cause allergic diseases (48).

Children born by vaginal delivery are colonized by bacteria found in the maternal vaginal and gastrointestinal microbiota, while newborn delivered by Caesarean section are colonized by bacteria present on the skin (51).

In order to determine possible differences between the skin of healthy individuals and that of subjects with the disorder, the skin microbiome was studied. The results reported that subjects with $\mathrm{AD}$ had a greater concentration of $S$. aureus compared to healthy subjects and that the composition of the skin microbiota underwent drastic changes as a result of corticosteroid therapy. In fact, during exacerbation of an atopic flare, the skin showed reduced bacterial biodiversity that was gradually repopulated as a result of corticosteroid therapy. Conversely, the most prevalent staphylococcal species on the skin of healthy controls belonged to the genus Epidermidis that, in association with other coagulase-negative staphylococci $(C o N S)$, can secrete antimicrobials that limit the overgrowth and biofilm formation of $S$. aureus (54).

It appears that not only bacterial species of the microbioma are implicated in the development of AD. Lunjani et al showed that fungal Malassezia DNA was detected in $90 \%$ of AD skin lesions and might contribute to the inflammatory process pathogenesis by secreting immunogenic proteins that induce the production of proinflammatory cytokines on keratinocytes (55).

\section{Controversies}

Trying to standardize data regarding the use of prebiotics and probiotics in $\mathrm{AD}$, we encountered tremendous variability of data. Literature abounds in conflicting data: studies regarding prophylactic and therapeutic applications, different types of strains and dosages, applications in young children up to 5 years of age and above, usage of probiotics alone, prebiotics alone or synbiotics combined. There are also conflicting data regarding the outcome of these studies, some proving a positive effect of prebiotics, probiotics or synbiotics and some showing no efficacy at all.

We performed a review of the most relevant articles regarding the use of these substances which were divided into those studying probiotics or prebiotics alone and a combination of the two, studies showing a positive effect and studies proving no efficacy at all. We tried to critically analyze those articles showing weak and strong points. 
Thus, Passeron et al published a double-blind prospective randomized study on the optimal combinations of probiotics and prebiotics (synbiotics), which could have efficacious results in the treatment of $\mathrm{AD}$, moderate and severe forms in children 2 years of age or older. The average age of the patients included in the study was 5.8 years. Initially, they included 48 patients divided into two groups, who received $1.2 \times 10^{9}$ colony-forming units of Lactobacillus rhamnosus Lcr35, plus a prebiotic or an identically prebiotic preparation alone three times a day for 3 months. The SCORAD score was initially calculated and had to be at least 15 , and it was again established to evaluate the efficiency of the treatment. The total SCORAD score mean in both groups was statistically significant after 3 months of treatment. The authors did not find any statistical differences between the two groups of treatment using the objective SCORAD score and total SCORAD or the total numbers of flares during the entire study or the mean numbers of flares in the month before the study and at the end of the treatment. Regarding topical treatments, the authors divided patient into three groups, according to what they were administered: topical steroids, calcineurin inhibitor ointments and emollients only. The authors did not find any statistically significant difference between the three groups regarding the total quantities of ointment used. The three groups formed regarding the ointment treatments were unequal; 34 patients in the first group, 11 in the second and only 3 in the third group. The study confirmed that the synbiotic treatment did not have better results than the prebiotics used alone. The small number of patients included in the study and the age of the patients included could also represent a weak point of the study, a higher efficiency having been stressed in children younger than two years of age. The comparison of prebiotics with synbiotics as approaches in the treatment of AD represents a strong aspect of the study, in comparison with studies that focus on probiotics or prebiotics alone (39).

The International Scientific Association for Probiotics and Prebiotics (ISAPP) convoked a board comprised of physiologists, nutritionists and microbiologists in May 2019, with the purpose of revising the 'synbiotics' definition, as well as their scope. They updated the definition to 'a mixture comprising live microorganisms and substrate(s) selectively utilized by host microorganisms that confers a health benefit on the host'. According to the board, by only defining synbiotics as a combination of probiotics and prebiotics would have suppressed the innovation of synbiotics, which are meant to work conjointly. In addition, the requirement that every element should meet the evidence, as well as dose specifications for both prebiotics and probiotics, could result in an impediment. Instead, the board made clear that a complementary synbiotic has not been designed so that its constituent parts work cooperatively while a synergistic synbiotic does not have to. A synbiotic whose substrate is used selectively by the co-administered microorganism constitutes a synergistic symbiotic (56).

According to research, the species belonging to the Lactobacillus, Bifidobacterium and Streptococcus genera represent the most used living microorganisms in the tested formulations. Generally, the substrate constituents are inulin, fructo-oligosaccharides or galacto-oligosaccharides; however, doses differ significantly, from as low as $100 \mathrm{mg}$ to as much as $10-15 \mathrm{~g}$ per day. For instance, in a double-blind randomized controlled trial (RCT), the consumption twice per day of a synbiotic composed of Lactobacillus casei 10 ( $\left.10^{9} \mathrm{CFU}\right)$, fructo-oligosaccharides (100 mg), Lactobacillus rhamnosus HS111 (10 $\left.{ }^{9} \mathrm{CFU}\right)$, Lactobacillus acidophilus 10 (10 $\left.{ }^{9} \mathrm{CFU}\right)$, Bifidobacterium bifidum ( $\left.10^{9} \mathrm{CFU}\right) 4$ days before and 10 days after surgery for periampullary neoplasms led to a reduced number of infections postoperatively. In addition, it resulted in fewer non-infectious complications, a shorter duration of antibiotic therapy, a reduced number of hospitalization days and lower mortality rate than in patients who received a placebo, namely sucrose (57). Even though such low doses would not be expected to be responsible for a prebiotic effect in complementary synbiotics, they could, theoretically, be sufficient to stimulate a cognate microorganism in a synergistic synbiotic formulation. In an RCT on 225 overweight and obese adults, a mixture of polydextrose (12 g per day) and Bifidobacterium animalis subsp. Lactis 420 led to a certain reduction in the body fat mass of $4.5 \%$, while the individual treatments produced no effects (58). Nonetheless, in this study, the use in a selective manner has not been established. Therefore, by itself, this research does not provide concluding proof that the combination tested was either a complementary or a synergistic synbiotic.

Aldaghi et al performed an RCT on 81 subjects with AD. The subjects were assigned to three groups at random. The synbiotic group received a dose of 5 drops/day of a synbiotic, besides the routine treatment. The vitamin D3 group received 1,000 units (IU) of vitamin D3 daily, aside from the routine treatment. According to the results, an effective way to reduce the severity of AD in infants is represented by multistrain synbiotics, alongside vitamin D3 supplements and routine treatments (59).

There are several RCT studies that have shown no efficacy of symbiotics in the outcome of AD. Dissanayake et al studied emollients vs. synbiotics in AD and showed that neither had any effect on reducing the progression of AD and food allergy at 1 year of age in a group of 459 children under 1 year of age (60).

In a study published by Isolauri et al, children younger than two years old with proven allergy to cow's milk had a better response to probiotics (61).

Different strains of prebiotics have been studied in order to manipulate the gut microbiome.

In a double-blind randomized placebo controlled trial, published by Weston et al, 56 children were included and then divided into two groups. In the first group, children received treatment with a probiotic $\left(1 \times 10^{9}\right.$ Lactobacillus fermentum VRI-033 PCC) two times per day for 8 weeks. In the second group, an equivalent volume of placebo (maltodextran) was administered during the same period of time. The children included in the study were aged between 6 and 18 months, with an average of 11.5 months, with moderate or severe AD. The SCORAD index was established to determine the efficacy of the treatment. An inclusion criterion in the study for the patients was having an SCORAD index $\geq 25$. Patient included were evaluated at week 0 when included, weeks 2 and 4 during the study and week 8 at the end of the study and at a later control, week 16 post-treatment. Topical corticosteroid treatment was continued during the study. Regarding the SCORAD index, the authors highlighted better improvement in the 
probiotic group compared to the placebo group at each week of the evaluation, with a reduction in the SCORAD index over time being statistically significant only in the probiotic group. The authors also analyzed the quality of life using the Dermatitis Family Impact Questionnaire (DFIQ), frequency of topical corticosteroid usage and parental impression of the intervention. The results were favorable in both groups regarding the DFIQ after treatment. Concerning the amount of topical corticosteroid applied, no significant difference was observed in any of the groups after treatment (62).

Every patient included in the study (62) was tested for total $\operatorname{IgE}$ and underwent an radioallergosorbent test (RAST), showing that total $\operatorname{IgE}$ was elevated in most patients, and RAST testing for specific IgE was positive to food mix in most of the patient, but they were less frequently positive to house dust mite allergen-specific IgE. Therefore, data on allergen avoidance regarding house dust mite may not be effective in controlling AD in children, as shown in a review published in 2020 (12). Bumbacea et al found a statistically significant difference in lower respiratory tract infections with a lower incidence of infections in the probiotic group, but no difference was seen regarding gastrointestinal symptoms or wheezing. One of the explanations invoked by the authors for the reduction of the SCORAD index in both groups was the fact that the behavior of the patients may have been altered by the inclusion in the study with a better compliance with previously prescribed treatment regimens and that the positive outcome in the placebo group may be explained by the natural evolution of the disease at this age. The follow-up of the patients until week 16 represents a strong aspect of the study, but the total number of patients included in the study was small, with only 56 patients being included (12).

The mechanisms proposed for probiotics consist of modifications in the innate immune system, such as the induction of regulatory $\mathrm{T}$ cell development (63) and alterations in Toll-like receptor (TLR) expression (64). Different probiotic strains may have varying effects. The role of Bifidobacterium or Lactobacillus supplements on TLR expression was assessed by Marlow et al in a study that used buccal smears sampled from 331 children. They showed that Lactobacillus was associated with 26 polymorphic TLR modifications that lowered the risk of eczema $(\mathrm{P}<0.02)$, as opposed to only two polymorphisms in those supplemented with Bifidobacterium (65).

In vitro, the prebiotics in human milk were found to act as decoy glycan receptors, which resulted in blocking certain parasites, viruses and bacteria from binding to epithelial cells (66-69). It appears that these prebiotics also affect directly the gene expression of epithelial cell surface glycans (70). In theory, the effects of reduced cell surface binding and improved glycan expression would help in the epithelial barrier function, which is proven to play a key role in the prevention of atopy. In vitro studies have proven that, alongside these indirect mechanisms, prebiotics seem to directly modulate immune responses to reduce IL-4 production, a known allergy mediator, in the lymphocytes of peanut allergic adults (71). An improved Th1 response, as well as a reduced Th2 (allergic) response to vaccination was found in mice supplemented with prebiotics (72). Moreover, in vitro, human milk prebiotics have been shown to reduce leukocyte rolling on TNF $\alpha$-activated human cells (73), in addition to platelet-neutrophil complex formation and neutrophil activation (74), which could possibly translate to diminished inflammatory responses.

In their review, Zhao et al evaluated the treatment efficacy of probiotics in children with AD by seven double-blinded randomized clinical trials, which included gathering 609 patient who received Lactobacillus. The outcome was favorable with a significant statistical difference in the SCORAD index. The authors did not include studies using synbiotics or a mixture of prebiotic and probiotics. Children included in the study were not older than 3 years of age. The SCORAD index was used as outcome at the end of the treatment. The authors showed that only preparations containing the Lactobacillus species were beneficial and that the duration of 8 weeks of treatment or less showed a statistically significant drop in the SCORAD index. In addition, the authors highlighted the treatment efficacy of the probiotic treatment when used in children of one year or less of age. The review also demonstrated that probiotic treatment in moderate to severe forms of the disorder was more effective than in mild forms. The authors stated that the results of the study may be explained by other studies that have shown lower bifidobacteria strains in the stools of children diagnosed with AD. Regarding the age of the patients, the authors explained that patients younger than 1 year of age might be less exposed to food components that act as allergens at elder ages (75).

A total of 43 patients diagnosed with moderate to severe forms of $\mathrm{AD}$, aged between 0 and 11 years who received 2 doses $\left(1 \times 10^{9} \mathrm{CFU} / \mathrm{sach}\right.$ t of $L$. salivarius LS01)/day during 8 weeks, followed by 1 dose/day during 8 weeks were included in an Italian study published by Niccoli et al. Patients continued to use emollients and/or topical steroid treatment if needed. Patients included in the study were evaluated at the beginning of the study and at $4,8,12,16$ weeks and 4 weeks after the end of the study at week 20. Objective SCORAD, SCORAD index and itch index were established to evaluate the outcome of the treatment. The authors concluded that after probiotic treatment, patients presented a significant statistical reduction in SCORAD and itch indexes and that effect persisted 1 month after the treatment with probiotic finished. However, the study gathered only a small cohort of patients and the authors pointed that a double-blind study was also required (76).

In a double-blind, placebo-controlled, crossover study published by Rosenfeldt et al, 43 patients aged between 1 to 13 years (mean age 5.2 years), received, during a period of 6 weeks, a mix of two probiotics Lactobacillus rhamnosus 19070-2 and Lactobacillus reuteri DSM 122460 at a dose of $10^{10}$ colony-forming units of each strain or an placebo preparation (mix of skimmed milk powder and dextrose anhydrate), two times a day. In the study, patients with moderate and severe AD were included. In order to establish the clinical severity of the eczema, patients were evaluated using the SCORAD score. Skin prick tests were performed for patients included in the study, as well as blood tests for serum IgE, serum eosinophil cationic protein and cytokines, such as IL-2, IL-4, IL-10 and INF- $\gamma$. Patients were than divided into two groups: group A received a placebo followed by active treatment; group B received active treatment followed by a placebo. Another repartition of the 43 patients included in the study was allergic and nonallergic patients. Allergic patients had a serum IgE lever higher than normal levels combined with positive skin prick test, elevated serum IgE levels with alimentary food component allergies, asthma, or allergic rhinoconjunctivitis. 
The personal evaluation of the patients regarding treatment was in favor of the active probiotic treatment with a statistically significant difference. Regarding the SCORAD index during active probiotic treatment, a lowering in the SCORAD index was seen, but with no statistically significant difference. A statistically significant difference during active probiotic treatment with impact on only one of the items of the SCORAD index (extent of the lesion) was seen only in allergic patients. The authors did not find any significant differences regarding topical corticosteroids use between active probiotic treatment and placebo. Concerning serum eosinophil cationic protein, a statistically significant difference was observed between active probiotic treatment and the placebo in favor of probiotics. No significant change was observed between the two groups regarding the cytokine levels. A possible weak point of the study might be the fact that the authors obtained a statistically significant difference only after splitting the SCORAD index. This study represents one of the few studies to use a mix of probiotics compared to a placebo (77).

\section{Conclusions}

In order to better understand the management of such a complex disorder as atopic dermatitis (AD), with major impact not only on the patients but also on their family, further studies concerning the use of probiotics and/or prebiotics in the treatment of children diagnosed with AD are still required. The main challenge is represented by the great heterogeneity of studies already published. Among these, some compare probiotic with prebiotics as single therapy or in association, as well as single strain vs. mixtures of strains of probiotics. There are also studies regarding various doses or the duration of treatment, as well as the duration of follow-up post treatment. We also observed a great diversity regarding the age of patients included in the studies, the severity of the disease and the inclusion or exclusion of patients with other allergic disease. A possible key solution would be the use of standard guidelines for study designs already proposed in the literature since 2010 by Shane et al (78).

In summary, the most studied probiotics were the strains of Lactobacilli and Bifidobacteria. The SCORAD index was used to measure the efficacy of the treatment. Most studies compared their results with a placebo group and the efficacy when seen in moderate to severe forms of AD in patients with other allergic diseases present. However, the results are difficult to be interpreted, as in many studies the authors suggest that the disease may have a tendency to improve in time in some groups of patients.

\section{Acknowledgements}

Not applicable.

\section{Funding}

No funding was received.

\section{Availability of data and materials}

All information included in this review is documented by relevant references.

\section{Authors' contributions}

ISF, DH, PL and SA performed the study of the literature data. DB, LMI, SC and RB critically revised the manuscript in light of the literature findings. All authors read and approved the final manuscript for publication.

\section{Ethics approval and consent to participate}

Not applicable.

\section{Patient consent for publication}

Not applicable.

\section{Competing interests}

The authors declare that they have no competing interests.

\section{References}

1. Sansotta N, Peroni DG, Romano S, Rugiano A, Vuilleumier P and Baviera G; Italian Society of Pediatric Allergy, Immunology (SIAIP), Microbiota Committee, Italy: The good bugs: The use of probiotics in pediatrics. Curr Opin Pediatr 31: 661-669, 2019.

2. Bantz SK, Zhu Z and Zheng T: The atopic march: Progression from atopic dermatitis to allergic rhinitis and asthma. J Clin Cell Immunol 5: 202, 2014.

3. Bumbăcea RS, Deaconu CG and Berghea EC: Management problems in severe chronic inducible urticaria: Two case reports. Exp Ther Med 18: 960-963, 2019.

4. Bumbacea R, Berghea E and Giurcaneanu C: Frequency of contact sensitisation in children with atopic dermatitis. Allergy 62 (Suppl 83): S319, 2007.

5. Eichenfield LF, Tom WL, Chamlin SL, Feldman SR, Hanifin JM, Simpson EL, Berger TG, Bergman JN, Cohen DE, Cooper KD, et al: Guidelines of care for the management of atopic dermatitis: Section 1. Diagnosis and assessment of atopic dermatitis. J Am Acad Dermatol 70: 338-351, 2014.

6. No authors listed: Worldwide variation in prevalence of symptoms of asthma, allergic rhinoconjunctivitis, and atopic eczema: ISAAC The International Study of Asthma and Allergies in Childhood (ISAAC) Steering Committee. Lancet 351: 1225-1232, 1998.

7. Rather IA, Bajpai VK, Kumar S, Lim J, Paek WK and Park YH: Probiotics and atopic dermtitis: An overview. Front Microbiol 7: 507, 2016.

8. Monti F, Agostini F, Gobbi F, Neri E, Schianchi S and Arcangeli F: Quality of life measures in Italian children with atopic dermatitis and their families. Ital J Pediatr 37: 59, 2011.

9. Chernyshov PV, Sampogna F, Pustišek N, Marinovic B, Manolache L, Suru A, Salavastru CM, Tiplica GS, Stoleriu G, Kakourou T, et al: Validation of the dermatology-specific proxy instrument the infants and toddlers dermatology quality of life. J Eur Acad Dermatol Venereol 33: 1405-1411, 2019.

10. Kim NY and Ji GE: Effects of probiotics on the prevention of atopic dermatitis. Korean J Pediatr 55: 193-201, 2012.

11. Solomon I, Ilie MA, Draghici C, Voiculescu VM, Caruntu C, Boda D and Zurac S: The impact of lifestyle factors on evolution of atopic dermatitis: An alternative approach. Exp Ther Med 17: 1078-1084, 2019

12. Bumbacea RS, Corcea SL, Ali S, Dinica LC, Fanfaret IS and Boda D: Mite allergy and atopic dermatitis: Is there a clear link? (Review). Exp Ther Med 20: 3554-3560, 2020.

13. Meneghin F, Fabiano V, Mameli C and Zuccotti GV: Probiotics and atopic dermatitis in children. Pharmaceuticals (Basel) 5: 727-744, 2012.

14. Bjorksten B: The intrauterine and postnatal environments. J Allergy Clin Immunol 104: 1119-1127, 1999.

15. Ro ADB, Simpson MR, R $\varnothing$ TB, Storr $\varnothing$ O, Johnsen R, Videm V and Øien T: Reduced Th22 cell proportion and prevention of atopic dermatitis in infants following maternal probiotic supplementation. Clin Exp Allergy 47: 1014-1021, 2017. 
16. Strachan DP: Hay fever, hygiene, and household size. BMJ 299: 1259-1260, 1989.

17. Strachan DP: Family size, infection and atopy: The first decade of the 'hygiene hypothesis'. Thorax 55 (Suppl 1): S2-S10, 2000.

18. Prescott SL, Macaubas C, Holt BJ, Snallacombe TB, Loh R, Sly PD and Holt PG: Transplacental priming of the human immune system to environmental allergens: Universal skewing of initial T cell responses toward the Th2 cytokine profile. J Immunol 160: 4730-4737, 1998.

19. Kopp MV, Zehle C, Pichler J, Szépfalusi Z, Moseler M, Deichmann K, Forster J and Kuehr J: Allergen-specific T cell reactivity in cord blood: The influence of maternal cytokine production. Clin Exp Allergy 31: 1536-1543, 2001.

20. Prescott SL, Macaubas C, Smallacombe T, Holt BJ, Sly PD and Holt PG: Development of allergen-specific T-cell memory in atopic and normal children. Lancet 353: 196-200, 1999.

21. Sudo N, Sawamura S, Tanaka K, Aiba Y, Kubo C and Koga Y: The requirement of intestinal bacterial flora for the development of an IgE production system fully susceptible to oral tolerance induction. J Immunol 159: 1739-1745, 1997.

22. Riedler J, Braun-Fahrlander C, Eder W, Schreuer M, Waser M, Maisch S, Carr D, Schierl R, Nowak D and von Mutius E; ALEX Study Team: Exposure to farming in early life and development of asthma and allergy: A cross-sectional survey. Lancet 358: $1129-1133,2001$

23. Taylor AL, Dunstan JA and Prescott SL: Probiotic supplementation for the first 6 months of life fails to reduce the risk of atopic dermatitis and increases the risk of allergen sensitization in high-risk children: A randomized controlled trial. J Allergy Clin Immunol 119: 184-191, 2007.

24. Williams H, Robertson C, Stewart A, Aït-Khaled N, Anabwani G, Anderson R, Asher I, Beasley R, Björkstén B, Burr M, et al: Worldwide variations in the prevalence of symptoms of atopic eczema in the International study of asthma and allergies in childhood. J Allergy Clin Immunol 103: 125-138, 1999.

25. Brandt EB and Sivaprasad U: Th2 cytokines and atopic dermatitis. J Clin Cell Immunol 2: 110, 2011.

26. Kubo A, Nagao K and Amagai M: Epidermal barrier dysfunction and cutaneous sensitization in atopic diseases. J Clin Invest 122: 440-447, 2012

27. Candela M, Rampelli S, Turroni S, Severgnini M, Consolandi C, De Bellis G, Masetti R, Ricci G, Pession A and Brigidi P: Unbalance of intestinal microbiota in atopic children. BMC Microbiol 12: 95, 2012

28. Penders J, Stobberingh EE, van den Brandt PA and Thijs C: The role of the intestinal microbiota in the development of atopic disorders. Allergy 62: 1223-1236, 2007.

29. Baker BS: The role of microorganisms in atopic dermatitis. Clin Exp Immunol 144: 1-9, 2006

30. Kunz B, Oranje AP, Labrèze L, Stalder JF, Ring J and Taïeb A: Clinical validation and guidelines for the SCORAD index: Consensus report of the European task force on atopic dermatitis. Dermatology 195: 10-19, 1997.

31. Guarner F and Schaafsma GJ: Probiotics. Int J Food Microbiol 39: 237-238, 1998.

32. Van Santvoort HC, Besselink MG, Timmerman HM, van Minnen LP, Akkermans LM and Gooszen HG: Probiotics in surgery. Surgery 143: 1-7, 2008.

33. Frei R, Akdis M and O'Mahony L: Prebiotics, probiotics, synbiotics, and the immune system: Experimental data and clinical evidence. Curr Opin Gastroenterol 31: 153-158, 2015.

34. Enomoto T, Sowa M, Nishimori K, Shimazu S, Yoshida A, Yamada K, Furukawa F, Nakagawa T, Yanagisawa N, Iwabuchi N, et al: Effects of bifidobacterial supplementation to pregnant women and infants in the prevention of allergy development in infants and on fecal microbiota. Allergol Int 63: 575-585, 2014.

35. Rusu E, Enache G, Cursaru R, Alexescu A, Radu R, Onila O, Cavallioti T, Rusu F, Posea M, Jinga M and Radulian G: Prebiotics and probiotics in atopic dermatitis. Exp Ther Med 18: 926-931, 2019.

36. Borchers AT, Selmi C, Meyers FJ, Keen CL and Gershwin ME: Probiotics and immunity. J Gastroenterol 44: 26-46, 2009.

37. Alvarez-Olmos MI and Oberhelman RA: Probiotic agents and infectious diseases: A modern perspective on a traditional therapy. Clin Infect Dis 32: 1567-1576, 2001.

38. Roberfroid M, Gibson GR, Hoyles L, McCartney AL, Rastall R, Rowland I, Wolvers D, Watzl B, Szajewska H, Stahl B, et al: Prebiotic effects: Metabolic and health benefits. Br J Nutr 104 (Suppl 2): S1-S63, 2010
39. Passeron T, Lacour JP, Fontas E and Ortonne JP: Prebiotics and synbiotics: Two promising approaches for the treatment of atopic dermatitis in children above 2 years. Allergy 61: 431-437, 2006.

40. Gibson GR and Roberfroid MB: Dietary modulation of the human colonic microbiota: Introducing the concept of prebiotics. J Nutr 125: 1401-1412, 1995

41. Maslowski KM, Vieira AT, Ng A, Kranich J, Sierro F, Yu D, Schilter HC, Rolph MS, Mackay F, Artis D, et al: Regulation of inflammatory responses by gut microbiota and chemoattractant receptor GPR43. Nature 461: 1282-1286, 2009.

42. De Preter V, Geboes K, Verbrugghe K, De Vuyst L, Vanhoutte T, Huys G, Swings J, Pot B and Verbeke K: The in vivo use of the stable isotope-labelled biomarkers lactose-[15N]ureide and [2H4]tyrosine to assess the effects of pro- and prebiotics on the intestinal flora of healthy human volunteers. Br J Nutr 92: 439-446, 2004.

43. Schley PD and Field CJ: The immune-enhancing effects of dietary fibres and prebiotics. Br J Nutr 87 (Suppl 2): S221-S230, 2002.

44. Moro G, Minoli I, Mosca M, Fanaro S, Jelinek J, Stahl B and Boehm G: Dosage-related bifidogenic effects of galactoand fructooligosaccharides in formula-fed term infants. J Pediatr Gastroenterol Nutr 34: 291-295, 2002

45. Haarman M and Knol J: Quantitative real-time PCR assays to identify and quantify fecal Bifidobacterium species in infants receiving a prebiotic infant formula. Appl Environ Microbiol 71: 2318-2324, 2005.

46. Schmelzle H, Wirth S, Skopnik H, Radke M, Knol J, Böckler HM, Brönstrup A, Wells J and Fusch C: Randomized double-blind study of the nutritional efficacy and bifidogenicity of a new infant formula containing partially hydrolyzed protein, a high beta-palmitic acid level, and nondigestible oligosaccharides. J Pediatr Gastroenterol Nutr 36: 343-351, 2003.

47. Kovacs Z, Benjamins E, Grau K, Ur Rehman A, Ebrahimi M and Czermak P: Recent developments in manufacturing oligosaccharides with prebiotic functions. Adv Biochem Eng Biotechnol 143: 257-295, 2014.

48. Blaser MJ: The microbiome revolution. J Clin Invest 124: 4162-4165, 2014.

49. Gerlich J, Benecke N, Peters-Weist AS, Heinrich S, Roller D, Genuneit J, Weinmayr G, Windstetter D, Dressel H, Range U, et al: Pregnancy and perinatal conditions and atopic disease prevalence in childhood and adulthood. Allergy 73: 1064-1074, 2018

50. Huang YJ, Marsland BJ, Bunyavanich S, O'Mahony L, Leung DY, Muraro A and Fleisher TA: The microbiome in allergic disease: Current understanding and future opportunities-2017 PRACTALL document of the American academy of allergy, asthma \& immunology and the European academy of allergy and clinical immunology. J Allergy Clin Immunol 139: 1099-1110, 2017.

51. Di Gangi A, Di Cicco ME, Comberiati P and Peroni DG: Go with your gut: The shaping of T-cell response by gut microbiota in allergic asthma. Front Immunol 11: 1485, 2020.

52. Maslowski KM and Mackay CR: Diet, gut microbiota and immune responses. Nat Immunol 12: 5-9, 2011.

53. Gray LE, O'Hely M, Ranganathan S, Sly PD and Vuillermin P. The maternal diet, gut bacteria, and bacterial metabolites during pregnancy influence offspring asthma. Front Immunol 8: 365 , 2017.

54. Kong HH, Oh J, Deming C, Conlan S, Grice EA, Beatson MA, Nomicos E, Polley EC, Komarow HD; NISC Comparative Sequence Program, et al: Temporal shifts in the skin microbiome associated with disease flares and treatment in children with atopic dermatitis. Genome Res 22: 850-859, 2012.

55. Lunjani N, Satitsuksanoa P, Lukasik Z, Sokolowska M, Eiwegger T and O'Mahony L: Recent developments and highlights in mechanisms of allergic diseases: Microbiome. Allergy 73: 2314-2327, 2018.

56. Swanson KS, Gibson GR, Hutkins R, Reimer RA, Reid G, Verbeke K, Scott KP, Holscher HD, Azad MB, Delzenne NM and Sanders ME: The International scientific association for probiotics and prebiotics (ISAPP) consensus statement on the definition and scope of synbiotics. Nat Rev Gastroenterol Hepatol 17: 687-701, 2020.

57. Sommacal HM, Bersch VP, Vitola SP and Osvaldt AB: Perioperative synbiotics decrease postoperative complications in periampullary neoplasms: A randomized, double-blind clinical trial. Nutr Cancer 67: 457-462, 2015. 
58. Stenman LK, Lehtinen MJ, Meland N, Christensen JE, Yeung N, Saarinen MT, Courtney M, Burcelin R, Lähdeaho ML, Linros J, et al: Probiotic with or without fiber controls body fat mass, associated with serum zonulin, in overweight and obese adults-randomized controlled trial. EBioMedicine 13: 190-200, 2016.

59. Aldaghi M, Tehrani H, Karrabi M, Abadi FS and Sahebkar M: The effect of multistrain synbiotic and vitamin D3 supplements on the severity of atopic dermatitis among infants under 1 year of age: A double-blind, randomized clinical trial study. J Dermatolog Treat 1-6, 2020 (Epub ahead of print).

60. Dissanayake E, Tani Y, Nagai K, Sahara M, Mitsuishi C, Togawa Y, Suzuki Y, Nakano T, Yamaide F, Ohno H and Shimojo N: Skin care and synbiotics for prevention of atopic dermatitis or food allergy in newborn infants: A 2 × 2 factorial, randomized, non-treatment controlled trial. Int Arch Allergy Immunol 180: 202-211, 2019.

61. Isolauri E, Arvola T, Sutas Y, Moilanen E and Salminen S: Probiotics in the management of atopic eczema. Clin Exp Allergy 30: 1604-1610, 2000 .

62. Weston S, Halbert A, Richmond P and Prescott SL: Effects of probiotics on atopic dermatitis: A randomised controlled trial. Arch Dis Child 90: 892-897, 2005.

63. Smits HH, Engering A, van der Kleij D, de Jong EC, Schipper K, van Capel TM, Zaat BA, Yazdanbakhsh M, Wierenga EA, van Kooyk Y and Kapsenberg ML: Selective probiotic bacteria induce IL-10-producing regulatory T cells in vitro by modulating dendritic cell function through dendritic cell-specific intercellular adhesion molecule 3-brabbing nonintegrin. J Allergy Clin Immunol 115: 1260-1267, 2005.

64. Lesiak A, Smolewski P, Sobolewska-Sztychny D, Sysa-Jedrzejowska A and Narbutt J: The role of T regulatory cells and Toll-like receptors 2 and 4 in atopic dermatitis. Scand J Immunol 76: 405-410, 2012.

65. Marlow G, Han DY, Wickens K, Stanley T, Crane J, Mitchell EA, Dekker J, Barthow C, Fitzharris P, Ferguson LR and Morgan AR: Differential effects of two probiotics on the risks of eczema and atopy associated with single nucleotide polymorphisms to Toll-like receptors. Pediatr Allergy Immunol 26: 262-271, 2015.

66. Bode L: The functional biology of human milk oligosaccharides. Early Hum Dev 91: 619-622, 2015.

67. Ruiz-Palacios GM, Cervantes LE, Ramos P, Chavez-Munguia B and Newburg DS: Campylobacter Jejuni binds intestinal $\mathrm{H}(\mathrm{O})$ antigen (Fuc alpha1, 2Gal beta1, 4GlcNAc), and fucosyloligosaccharides of human milk inhibit its binding and infection. J Biol Chem 278: 14112-14120, 2003.

68. Manthey CF, Autran CA, Eckmann L and Bode L: Human milk oligosaccharides protect against enteropathogenic Escherichia. coli attachment in vitro and EPEC colonization in suckling mice. J Pediatr Gastroenterol Nutr 58: 167-170, 2014.
69. Jantscher-Krenn E, Lauwaet T, Bliss LA, Reed SL, Gillin FD and Bode L: Human milk oligosaccharides reduce Entamoeba histolytica attachment and cytotoxicity in vitro. Br J Nutr 108: 1839-1846, 2012.

70. Angeloni S, Ridet JL, Kusy N, Gao H, Crevoisier F, Guinchard S, Kochhar S, Sigrist H and Sprenger N: Glycoprofiling with micro-arrays of glycoconjugates and lectins. Glycobiology 15: 31-41, 2005

71. Eiwegger T, Stahl B, Haidl P, Schmitt J, Boehm G, Dehlink E, Urbanek R and Szépfalusi Z: Prebiotic oligosaccharides: In vitro evidence for gastrointestinal epithelial transfer and immunomodulatory properties. Pediatr Allergy Immunol 21: 1179-1188, 2010.

72. Vos AP, Haarman M, van Ginkel JW, Knol J, Garssen J, Stahl B, Boehm G and M'Rabet L: Dietary supplementation of neutral and acidic oligosaccharides enhances Th1-dependent vaccination responses in mice. Pediatr Allergy Immunol 18: 304-312, 2007.

73. Bode L, Kunz C, Muhly-Reinholz M, Mayer K, Seeger W and Rudloff S: Inhibition of monocyte, lymphocyte, and neutrophil adhesion to endothelial cells by human milk oligosaccharides. Thromb Haemost 92: 1402-1410, 2004.

74. Bode L, Rudloff S, Kunz C, Strobel S and Klein N: Human milk oligosaccharides reduce platelet-neutrophil complex formation leading to a decrease in neutrophil beta 2 integrin expression. J Leukoc Biol 76: 820-826, 2004.

75. Zhao M, Shen C and Ma L: Treatment efficacy of probiotics on atopic dermatitis, zooming in on infants: A systematic review and meta-analysis. Int J Dermatol 57: 635-641, 2018

76. Niccoli AA, Artesi AL, Candio F, Ceccarelli S, Cozzali R, Ferraro L, Fiumana D, Mencacci M, Morlupo M, Pazzelli P, et al: Preliminary results on clinical effects of probiotic Lactobacillus. salivarius LSO1 in children affected by atopic dermatitis. J Clin Gastroenterol 48 (Suppl 1): S34-S36, 2014.

77. Rosenfeldt V, Benfeldt E, Nielsen SD, Michaelsen KF Jeppesen DL, Valerius NH and Paerregaard A: Effect of probiotic lactobacillus strains in children with atopic dermatitis. J Allergy Clin Immunol 111: 389-395, 2003.

78. Shane AL, Cabana MD, Vidry S, Merenstein D, Hummelen R, Ellis CL, Heimbach JT, Hempel S, Lynch SV, Sanders ME and Tancredi DJ: Guide to designing, conducting, publishing and communicating results of clinical studies involving probiotic applications in human participants. Gut Microbes 1: 243-253, 2010.

This work is licensed under a Creative Commons Attribution-NonCommercial-NoDerivatives 4.0 International (CC BY-NC-ND 4.0) License. 